\title{
Luteolin reduces obesity-associated insulin resistance in mice by activating AMPK $\alpha 1$ signalling in adipose tissue macrophages
}

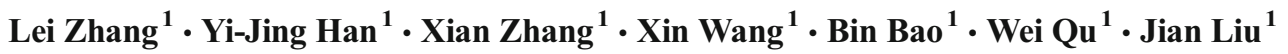

Received: 16 March 2016 / Accepted: 8 June 2016 / Published online: 4 July 2016

(C) Springer-Verlag Berlin Heidelberg 2016

\begin{abstract}
Aims/hypothesis Inflammatory polarisation of adipose tissue macrophages (ATMs) plays a critical role in the development of obesity-associated metabolic diseases such as insulin resistance and diabetes. Our previous study indicated that dietary luteolin (LU) could prevent the establishment of insulin resistance in mice fed a high-fat diet (HFD). Here, we further investigated the effects of $\mathrm{LU}$, which is a natural flavonoid, on pre-established insulin resistance and obesityassociated ATM polarisation in mice.

Methods Five-week-old C57/BL6 mice were fed on a low-fat diet or HFD for 20 weeks, with some mice receiving supplementation with $0.01 \%$ LU from weeks 1 or 10 of the HFD to assess the actions of LU on insulin resistance and ATM polarisation. Furthermore, the role of LU in metabolicdysfunction-associated macrophage phenotypes was investigated in vitro.

Results Dietary LU supplementation, either for 20 weeks or from weeks 10 to 20 of an HFD, significantly improved insulin resistance in HFD-fed mice. In addition, inflammatory macrophage infiltration and polarisation were suppressed in mouse epididymal adipose tissues. Furthermore, LU treatment directly reversed lipopolysaccharide-stimulated and metabolism-regulated molecules, and induced inflammatory polarisation in mouse RAW264.7 cells and peritoneal cavity
\end{abstract}

Electronic supplementary material The online version of this article (doi:10.1007/s00125-016-4039-8) contains peer-reviewed but unedited supplementary material, which is available to authorised users.

Jian Liu

liujian509@hfut.edu.cn

School of Biotechnology and Food Engineering, Hefei University of Technology, 193 Tunxi Road, Hefei, Anhui 230009, People's

Republic of China resident macrophages. Finally, using the selective AMPactivated protein kinase (AMPK) inhibitor compound $\mathrm{C}$ and Ampkal (also known as Prkaal) silencing with siRNA, we found that $L U$ activated AMPK $\alpha 1$ in macrophages to inhibit their inflammatory polarisation and enhanced insulin signals in adipocytes that were stimulated with macrophageconditioned media.

Conclusions/interpretation Dietary LU ameliorated insulin resistance in diet-induced obese mice by promoting $\mathrm{AMPK} \alpha 1$ signalling in ATMs.

Keywords Adipose tissue macrophage $\cdot$ AMPK $\alpha 1 \cdot$ Insulin resistance $\cdot$ Luteolin $\cdot$ Polarisation

$\begin{array}{ll}\text { Abbreviations } \\ \text { ABCA1 } & \text { ATP-binding cassette sub-family A member 1 } \\ \text { AICAR } & \begin{array}{l}\text { 5-Aminoimidazole-4-carboxamide 1- } \beta \text {-D- } \\ \text { ribofuranoside }\end{array} \\ \text { AMPK } & \text { AMP-activated protein kinase } \\ \text { ATM } & \text { Adipose tissue macrophage } \\ \text { CM } & \text { Conditioned media } \\ \text { DIO } & \text { Diet-induced obesity } \\ \text { EAT } & \text { Epididymal adipose tissue } \\ \text { HFD } & \text { High-fat diet } \\ \text { LFD } & \text { Low-fat diet } \\ \text { LPS } & \text { Lipopolysaccharide } \\ \text { LU } & \text { Luteolin } \\ \text { MMe } & \text { Metabolic activation macrophage } \\ \text { PCM } & \text { Peritoneal cavity resident macrophage } \\ \text { PIG } & \text { Palmitate insulin and glucose } \\ \text { SiRNA } & \text { Small interfering RNA } \\ \text { SVF } & \text { Stromal vascular fraction }\end{array}$




\section{Introduction}

Obesity, of which there is a worldwide epidemic, is fundamentally caused by a long-term energy imbalance. During the development of diet-induced obesity (DIO), overnutrition leads to adipocyte hypertrophy and progressive inflammatory cell infiltration into adipose tissues. Hypertrophic adipocytes and inflammatory cells secrete various proinflammatory cytokines and promote adipose tissue and systemic inflammation, finally resulting in insulin resistance and type 2 diabetes [1-3]. However, not every obese individual suffers from insulin resistance and type 2 diabetes, and not all body fat contributes equally to these common metabolic diseases. When responding to a high-fat diet (HFD), visceral adipose tissues undergo a greater degree of inflammatory cell recruitment and higher tissue inflammation compared with subcutaneous adipose tissues. Therefore, abdominal obesity and visceral fat, but not subcutaneous fat, are associated with a high risk of metabolic diseases [4-6].

Of all the immune cells, adipose tissue macrophages (ATMs) have been paid a great deal of attention and are regarded as critical to the development of obesity-associated inflammation and insulin resistance [7,8]. Epididymal adipose tissue (EAT) has been reported to be the most commonly used visceral fat depot in mouse studies, and its macrophage infiltration is most severe during the development of DIO [9]. In the lean state, EAT macrophages are mainly alternatively activated (M2) macrophages, which are characterised by CD206 and arginase 1 . M2 cells produce anti-inflammatory cytokines such as IL-10 and IL-1 receptor antagonist, and maintain insulin sensitivity [10]. In contrast, classically activated (M1) macrophages express CD11c and nitric oxide synthase 2, and predominate in EATs of obese animals. These cells produce and secrete high levels of proinflammatory cytokines such as TNF- $\alpha$ and IL-6, resulting in insulin resistance [11, 12]. Although this switch of M2 to M1 macrophages has been considered to be responsible for obesity-associated metabolic complications [13], ATM polarisation is more complex in obese states. Recently, Kratz et al used a mixture of palmitate, insulin and glucose (PIG) to produce a 'metabolic activation' macrophage (MMe) and reported the existence of this phenotype in EATs of obese mice [14]. This macrophage phenotype overexpresses the proinflammatory cytokines TNF- $\alpha$, IL- $1 \beta$ and IL-6, but not M1 macrophage surface markers [14].

Luteolin (LU) is a natural flavonoid that is found in many edible plants, including peppers, parsley, spinach, carrots and celery. We have previously reported that a low-dose dietary supplement of LU could block the establishment of insulin resistance in HFD-fed mice [15]. In addition, dietary LU reduced macrophage recruitment and the expression of proinflammatory cytokines in EATs in the mice. Moreover, several in vitro studies have reported that LU suppresses the production of the proinflammatory cytokines TNF- $\alpha$ and IL-6 in macrophages [16-18]. However, it is not clear whether LU affects pre-established insulin resistance and obesity-associated ATM polarisation in mice.

AMP-activated protein kinase (AMPK) is a member of the metabolite-sensing protein kinase family and a crucial metabolic and inflammatory regulator $[19,20]$. The catalytic subunit AMPK $\alpha 1$ is mainly expressed in macrophages and suppresses associated proinflammatory responses and polarisation [21, 22]. Previous studies have reported that LU can enhance the phosphorylation of AMPK in HepG2 cells [23] and 3T3-L1 adipocytes [24]. Therefore, the functional correlation between LU and AMPK $\alpha 1$ signalling in ATM inflammation and polarisation should be assessed.

The aim of this study was to evaluate whether LU ameliorated pre-established insulin resistance by inhibiting obesity-associated ATM polarisation in mice.

\section{Methods}

Animals Three-week-old male C57BL/6 mice were purchased from Vital River Laboratory Animal Technology Co. (Beijing, China) and housed in ventilated cages within a pathogen-free barrier facility that maintained a $12 \mathrm{~h}$ light: $12 \mathrm{~h}$ dark cycle. A total of 16 mice were fed an HFD from the age of 5 weeks. At 10 weeks, these mice were randomly divided into two groups: (1) an HFD group $(n=8)$, in which the animals were continued on the HFD; and (2) an HFD + LU10 group $(n=8)$, in which the animals were switched onto an HFD supplemented with $0.01 \%$ LU (Huayi Biotechnology, Shanghai, China). In addition, a further eight 5 -week-old mice were fed on a low-fat diet (LFD group), and eight 5-week-old mice were fed on an HFD supplemented with $0.01 \%$ LU for 20 weeks (HFD + LU20 group). The detailed contents of all of the diets are shown in the electronic supplementary material (ESM) Table 1.

The mice were weighed every week. At 15 and 25 weeks of age, the mice were fasted overnight and a GTT and insulin tolerance test were performed as previously described [15]. At 25 weeks of age, all mice were killed with $\mathrm{CO}_{2}$ and their blood and adipose tissues were harvested and stored at $-80^{\circ} \mathrm{C}$, as previously described [25]. All experimental procedures were approved by the Standing Committee on Animals of Hefei University of Technology.

Protein extraction and western blot analysis Protein extraction and immunoblots were performed as previously described [25]. See ESM Methods.

Total RNA isolation and quantitative real-time PCR Quantitative real-time PCR was used to determine the relative expression levels of mRNAs. See ESM Methods. 
Immunohistochemistry and ELISA Immunohistochemistry stains and ELISA were prepared as previously described [15]. See ESM Methods.

EAT stromal vascular fraction (SVF) isolation Stromal vascular cells were isolated as previously described [26], with some minor modifications. See ESM Methods.

Flow cytometry analysis Flow cytometry analysis was conducted to determine macrophage polarisation. See ESM Methods.

Small interfering (si)RNA siRNA was used to silence Ampkal (also known as Prkaa1). See ESM Methods.

Cell culture and treatment RAW264.7 macrophages were purchased from the Cell Culture Center of Peking Union Medical College (Beijing, China) and cultured in low D-glucose (1 g/1) DMEM supplemented with 10\% FBS. Peritoneal cavity cells were harvested from 8 -week-old male C57BL/6 mice. Using APC anti-mouse F4/80 antibody (BioLegend, San Diego, CA, USA), PCMs were sorted using a MoFlo XDP flow cytometer (Beckman Coulter, Fullerton, CA, USA) and cultured in low D-glucose (1 g/l) DMEM supplemented with $10 \%$ FBS.

RAW264.7 cells and PCMs were planted in 24-well plates and pretreated with vehicle, $20 \mu \mathrm{mol} / 1 \mathrm{LU}$ or $2 \mathrm{mmol} / 1$ of the AMPK $\alpha 1$ activator AICAR (5-aminoimidazole-4carboxamide 1- $\beta$-D-ribofuranoside; Sigma-Aldrich, St. Louis, MO, USA) for $12 \mathrm{~h}$. To examine the effects of LU on macrophage M1 polarisation, the cells were stimulated with $100 \mathrm{ng} / \mathrm{ml}$ LPS (Sigma-Aldrich) according to previously published reports $[14,25]$. The MMe phenotype polarisation method was performed according to the procedures described by Kratz et al [14]. These cells were treated with PIG (100 $\mu \mathrm{mol} / 1$ palmitate, $10 \mathrm{nmol} / \mathrm{l}$ insulin and $30 \mathrm{mmol} / \mathrm{l}$ glucose). In addition, compound C ( $1 \mu \mathrm{mol} / \mathrm{l})$ was added as an AMPK inhibitor 30 min before LU or AICAR treatment.

Following the method of Ceppo et al [27], with minor modifications, a series of conditioned media (CM) were produced from LPS- and PIG-stimulated RAW264.7 cells and PCMs. Briefly, LU or/and AMPK inhibitor or siRNA was used to treat the macrophages, as described above. The pretreated macrophages were then stimulated with LPS or PIG. After $3 \mathrm{~h}$, the macrophages were washed and incubated in fresh culture medium for $24 \mathrm{~h}$.

3T3-L1 pre-adipocytes were purchased from the Cell Culture Center of Peking Union Medical College and differentiated into adipocytes as previously described [28]. The differentiated adipocytes were cultured in the above-mentioned macrophage CM for $24 \mathrm{~h}$, followed by stimulation with $1 \mathrm{nmol} / 1$ insulin for $7 \mathrm{~min}$. The cells were then lysed for insulin signalling analysis.
Statistical analysis All data are presented as means \pm SEM. One-way ANOVAs with Duncan's post hoc tests were used for mouse assays. Two-tailed Student's $t$ tests were used for cell assays. $p<0.05$ was considered as statistically significant.

\section{Results}

Dietary LU not only prevented the establishment of diet-induced insulin resistance, but also reversed preestablished insulin resistance To confirm the effects of LU on the establishment of diet-induced insulin resistance, 5-week-old male mice were fed with an LFD, HFD or HFD supplemented with $0.01 \%$ LU. After 10 weeks, the mice fed on an HFD had gained significantly more body weight than those fed with an LFD (Fig. 1a). The GTT and insulin tolerance test revealed that HFD-fed mice had lower glucose tolerance and insulin sensitivity than LFD-fed mice (ESM Fig. 1), suggesting that diet-induced insulin resistance was established. Supporting our previous results, dietary LU remarkably reduced HFD-induced body weight gain (Fig. 1a) and insulin resistance (ESM Fig. 1) in mice.

To test whether dietary LU also reversed pre-established insulin resistance, the mice fed with an HFD were divided into an HFD group and an HFD + LU10 group. The remaining mice (i.e. those in the LFD and HFD + LU20 groups) continued on their respective diets. As expected, the HFD mice continued to gain body weight (Fig. 1a) and had greater adipose tissue weight (ESM Fig. 2a, b), lower glucose tolerance (Fig. 1b) and lower insulin sensitivity (Fig. 1c) compared with the LFD group. Furthermore, the body weight and insulin resistance of the HFD + LU20 group were significantly improved compared with those of the HFD group. Intriguingly, glucose homeostasis (Fig. 1b) and insulin sensitivity (Fig. 1c) were also ameliorated in the HFD + LU10 group, although the body weight (Fig. 1a) and adipose tissue weight (ESM Fig. 2a, b) of these animals were not affected by dietary LU. During 20 weeks of intervention, dietary LU did not affect food intake among the HFD-fed mice (ESM Fig. 2c).

Insulin can promote Akt phosphorylation to regulate adipose tissue glucose uptake and systemic glucose homeostasis. Along with improvements in insulin resistance, mice in the HFD + LU20 and HFD + LU10 groups had significantly more robust Akt phosphorylation in their EATs, similar to those in LFD group (Fig. 1d). Adipose tissue inflammation directly suppresses insulin signalling to develop insulin resistance. Therefore, we further looked at several important proinflammatory cytokines involved in obesityassociated insulin resistance, including TNF- $\alpha$, IL- 6 and monocyte chemoattractant protein 1. Dietary LU downregulated the EAT mRNA expression (Fig. 1e) and serum levels (Fig. 1f) of these cytokines in HFD-fed mice, 
Fig. 1 Five-week-old mice were fed on an LFD (white circles and hatched bars) or HFD (black circles and black bars) for 20 weeks. In addition, mice of the same age were fed with an HFD supplemented with $0.01 \% \mathrm{LU}$ during weeks 10-20 (HFD + LU10, grey triangles and grey bars) or weeks 1-20 (HFD + LU20, white squares and white bars) of the HFD. Body weight (a), and GTT (b) and insulin tolerance test (c) results at age 25 weeks. (d) Immunoblots for phospho-Akt (Ser473) and total Akt in EATs and quantification of phosphorylated Akt to total Akt proinflammatory cytokine mRNA levels in EATs (e) and proinflammatory mediators in serum (f). All data are presented as means \pm SEM, $n=8$ per group, one-way ANOVA with Duncan's post hoc test relative to HFD; ${ }^{*} p<0.05,{ }^{*} p<0.01$ a

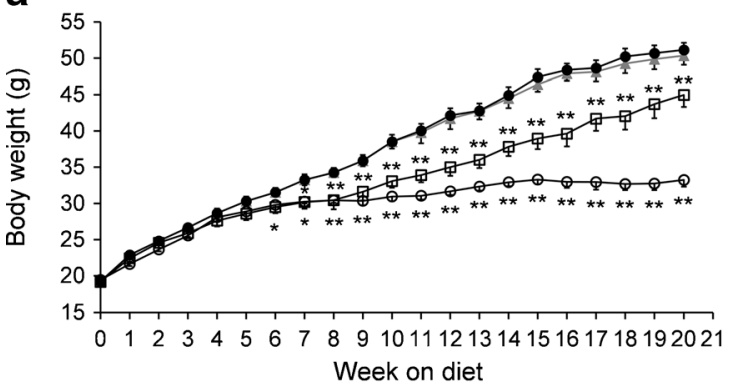

b

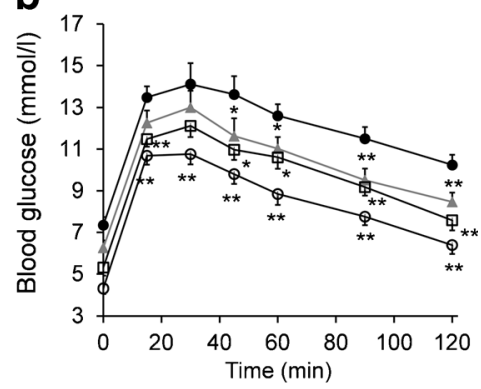

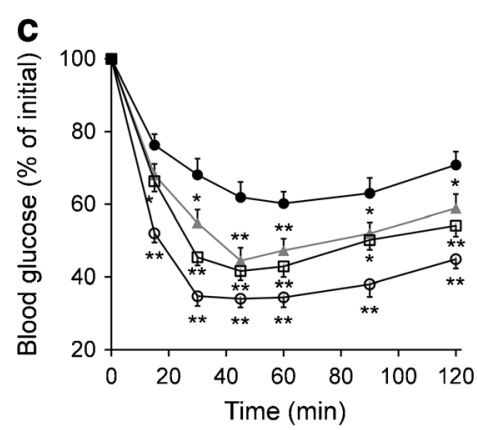

e

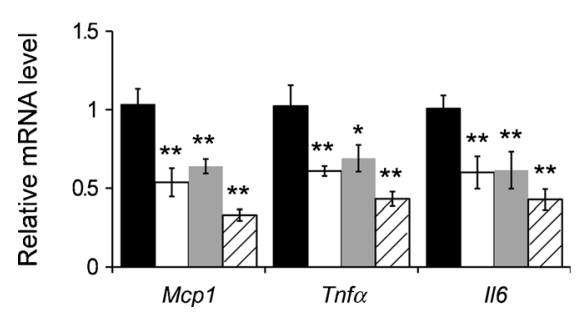

d
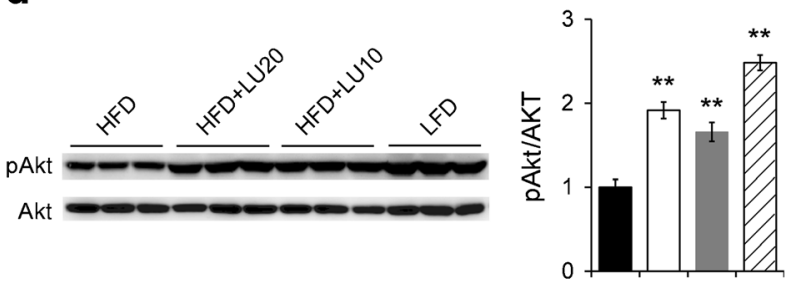

suggesting a decline in adipose tissue and systemic inflammation. In conclusion, dietary LU not only resisted the establishment of diet-induced insulin resistance, but also ameliorated pre-established insulin resistance in HFD-fed mice.

\section{Dietary LU abated HFD-induced macrophage infiltration into EATs Macrophages are the most abundant immune cells and the primary contributors to inflammation in adipose tissues in DIO mice. We used flow cytometry (Fig. 2a, ESM Fig. 3c) and macrophage-specific MAC-2 immunostaining (ESM Fig. 3a, b) to analyse EAT macrophage levels. The results indicated inhibition of macrophage infiltration by dietary LU.}

\section{Dietary LU reversed obesity-associated ATM polarisation} Given ATM polarisation is important in obesity-associated adipose tissue inflammation and insulin resistance, we further investigated EAT macrophage subsets. Flow cytometry showed that an HFD elevated the percentages of $\mathrm{CD} 11 \mathrm{c}^{+} \mathrm{F} 4 /$ $80^{+} \mathrm{M} 1$ macrophages and lowered those of $\mathrm{CD} 206^{+} \mathrm{F} 4 / 80^{+}$ M2 macrophages in EATs (Fig. 2a-d). Dietary LU markedly abolished M1 macrophage polarisation and enlarged the percentage of M2-type macrophages, resulting in a decline of the M1/M2 ratio in EATs in both the HFD + LU20 and HFD + LU10 groups (Fig. 2a-d). Recently, MMe was identified as a novel inflammatory macrophage phenotype in the adipose tissues of obese humans and mice. In this study, we also detected expression of the MMe markers $C d 36$ and Plin2 in EATs. An HFD resulted in the enhanced expression of these markers in EATs, while dietary LU abrogated the actions (Fig. 2e, f). These results suggest that dietary LU reverses both M1 and MMe polarisation in EATs in HFD-fed mice.

\section{LU treatment directly inhibited M1 and MMe polarisation} Since dietary LU inhibited obesity-associated ATM polarisation (Fig. 2), we attempted to investigate whether LU directly affects inflammatory macrophage polarisation, including LPS-stimulated M1 and PIG-induced MMe polarisation. As expected, the classic M1 mediator LPS and metabolic activation by PIG elevated the expression of the proinflammatory cytokine genes $M c p 1$ (also known as $C c l 2$ ), Tnf- $a$ (also known as Tnf) and Il-6 in RAW264.7 cells and PCMs (ESM Fig. 4). LU (5-20 $\mu \mathrm{mol} / \mathrm{l})$ downregulated the expression of these genes in a dose-dependent manner (ESM Fig. 4). 
Fig. 2 Dietary LU inhibited obesity-associated ATM polarisation in EATs. (a) Using flow cytometry, $\mathrm{F} 4 / 80^{+} \mathrm{CD} 11 \mathrm{c}^{+}$ M1-type and $\mathrm{F} 4 / 80^{+} \mathrm{CD} 206^{+} \mathrm{M} 2$ type macrophages from SVF cells in EATs were analysed. The percentage of M1- (b) and M2type (c) macrophages in total SVF cells and the ratio of M1 to M2 cells (d). mRNA levels of the MMe markers $C d 36$ (e) and Plin 2 (f) were quantified using quantitative real-time PCR. Black bars, HFD; white bars, HFD + LU20; grey bars, HFD + LU10; hatched bars, LFD. All data are presented as means \pm SEM, $n=8$ per group, one-way ANOVA with Duncan's post hoc test relative to HFD $;{ }^{*} p<0.05,{ }^{* *} p<0.01$. SSC, side scatter
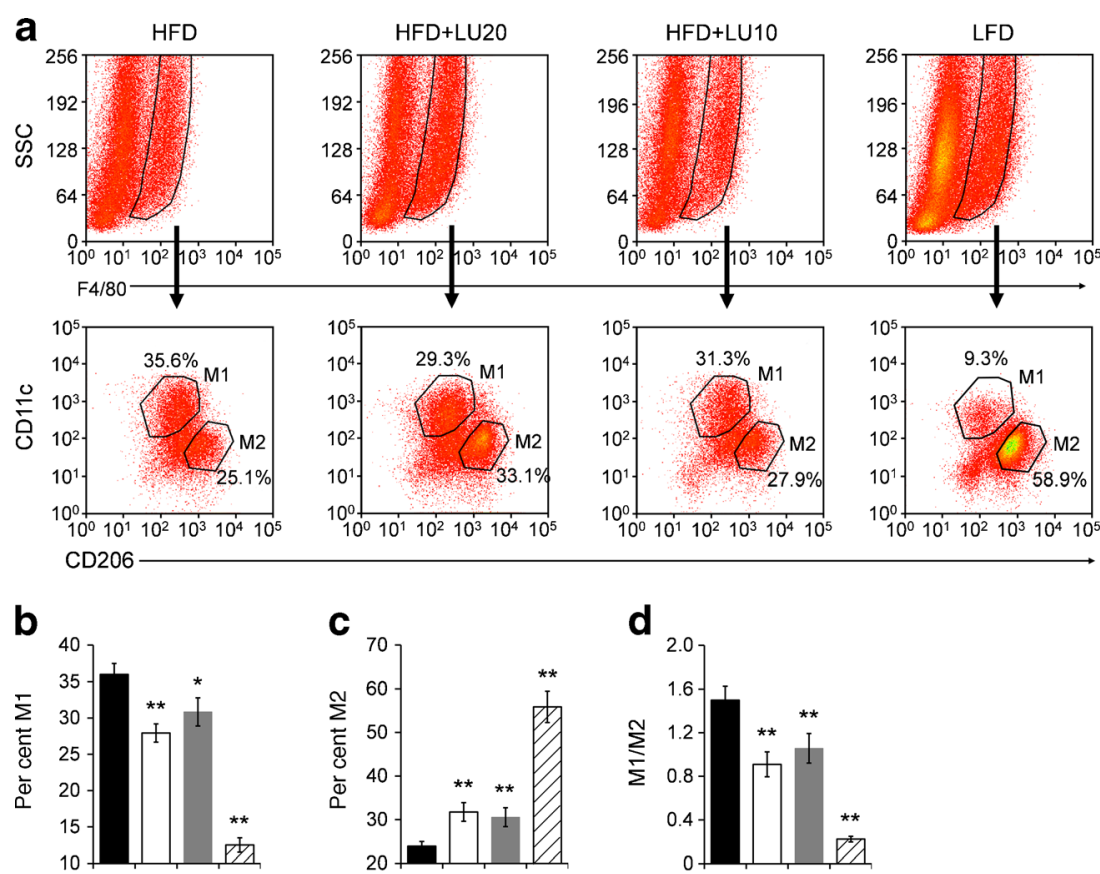

C

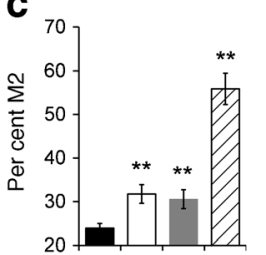

d

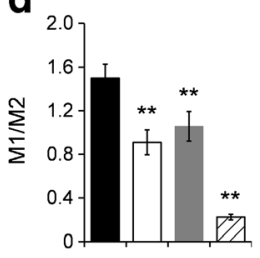

f
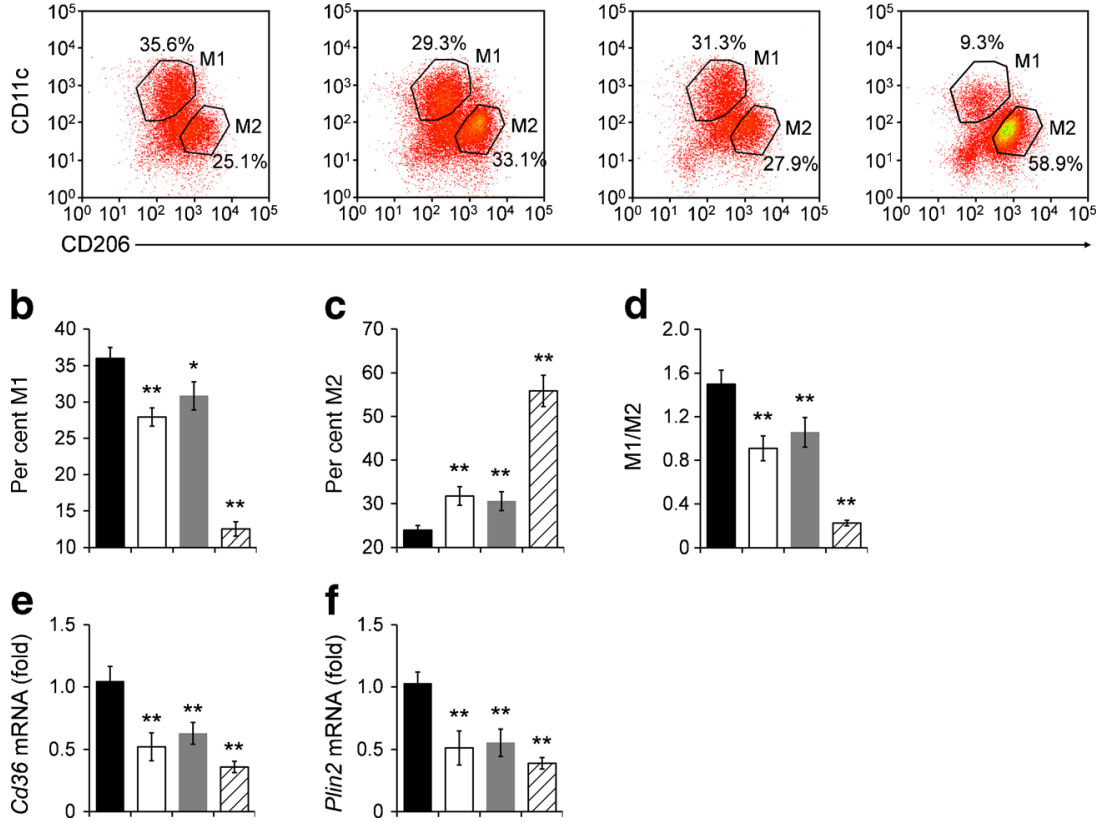

\section{西}

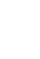

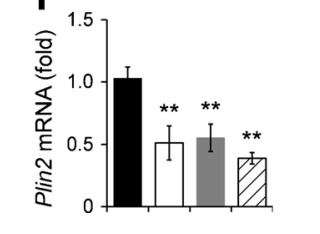

LU activated AMPK $\alpha 1$ signalling to reduce inflammatory macrophage polarisation AMPK is an important nutrient sensor and inflammatory suppressor. In macrophages, AMPK $\alpha 1$ signalling critically regulates metabolism-related inflammation and polarisation. To explore the involvement of AMPK $\alpha 1$ signalling in LU-associated reductions in ATM polarisation and inflammation, we first detected phosphorylated AMPK $\alpha 1$ levels in mouse EATs. Compared with mice in the HFD group, those in the LFD, HFD + LU10 and HFD + LU20 groups had obviously higher AMPK $\alpha 1$ phosphorylation (Fig. 5a), implying that dietary LU might suppress inflammatory ATM polarisation by activating AMPK $\alpha 1$ signalling.

In LPS-stimulated (Fig. 5b) and PIG-stimulated (Fig. 5c) conditions, both LU and the AMPK activator AICAR significantly reversed the inhibitory effects of the proinflammatory reagents on AMPK $\alpha 1$ phosphorylation in RAW264.7 macrophages and PCMs. Similarly, the selective AMPK inhibitor compound $\mathrm{C}$ abated AMPK $\alpha 1$ activation by LU and AICAR (Fig. 5b, c). Furthermore, both LU and AICAR treatment also counteracted LPS- and PIG-induced overexpression of the proinflammatory cytokine genes Mcp 1, Tnf- $a$ and Il-6 in RAW264.7 cells and PCMs (ESM Fig. 5). Polarisation-associated alterations in macrophage markers, including the M1 marker Nos2, the M2 marker Arg1 (Fig. 6a-d) and the MMe markers Cd36 and Plin2 and Plin2 in the macrophages (Fig. 4e-h). 
Fig. 3 LU repressed LPSinduced M1 macrophage polarisation. mRNA levels of the M1 marker Nos2 and the M2 marker Arg1 were detected in RAW264.7 cells (a, b) and PCMs (c, d). Expression of the M1-type macrophage markers CD38 and CD274 was detected using flow cytometry in RAW264.7 cells (e, f) and PCMs (g, h). Grey areas, control; red lines, LPS; blue lines, LPS + LU. All data are presented as means $\pm \mathrm{SEM}, n=4$ per group, two-tailed Student's $t$ test relative to LPS treatment; ${ }^{*} p<0.05$, $* * p<0.01$

Fig. 4 LU suppressed PIGinduced MMe polarisation. Expression of the MMe markers CD36 and ABCA1 was detected by flow cytometry in RAW264.7 cells (a, b) and PCMs (c, d). mRNA levels of the MMe markers $\mathrm{Cd} 36$ and Plin2 in RAW264.7 cells (e, f) and PCMs $(\mathbf{g}, \mathbf{h})$ were quantified using quantitative real-time PCR. Grey areas, control; red lines, LPS; blue lines, LPS + LU. All data are presented as means \pm SEM, $n=4$ per group, two-tailed Student's $t$ test relative to PIG treatment; ${ }^{*} p<0.05, * * p<0.01$
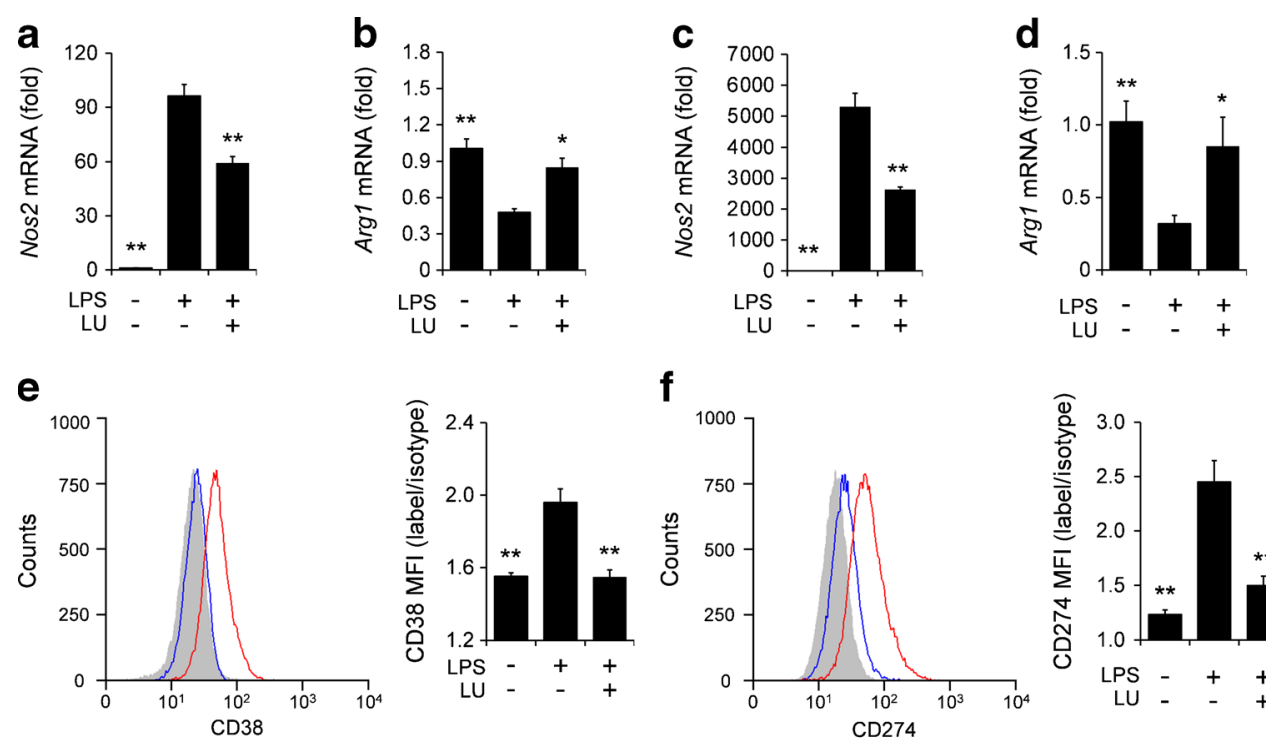

f
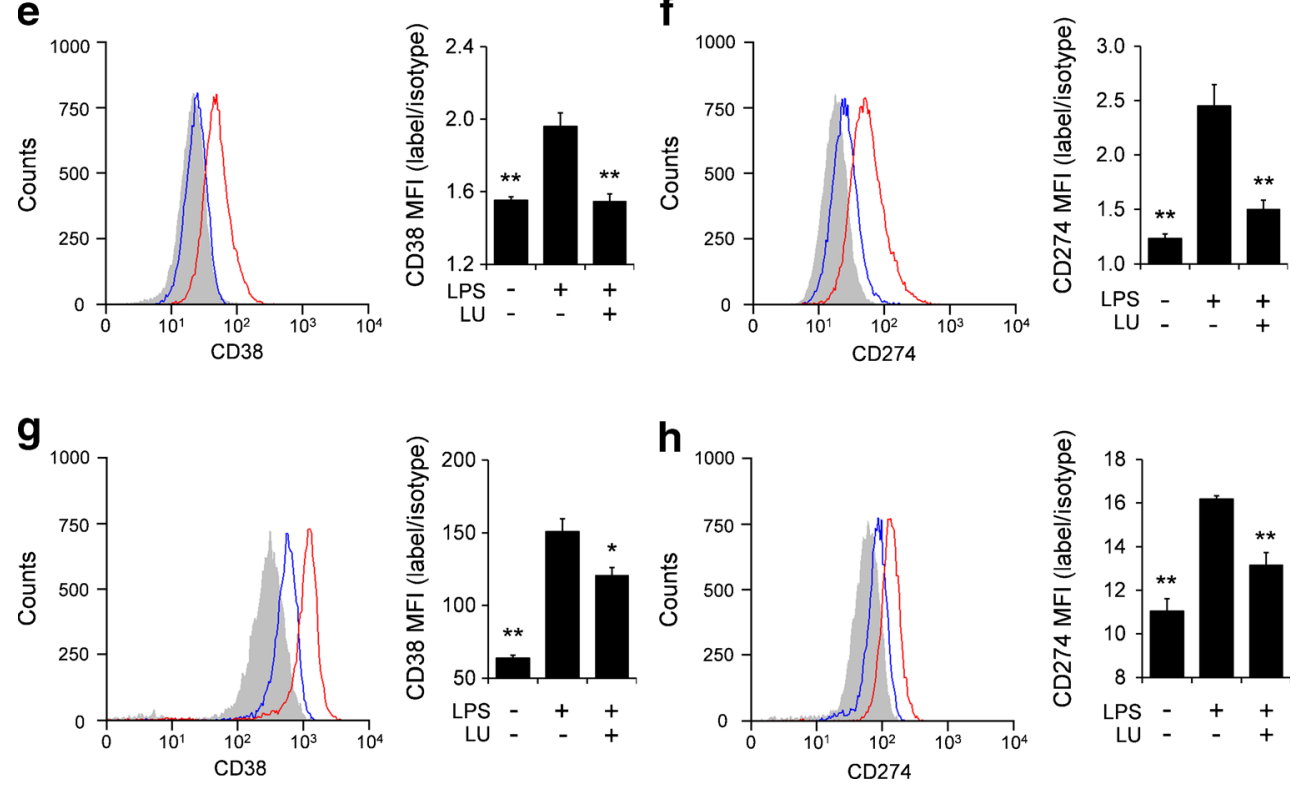

a


b
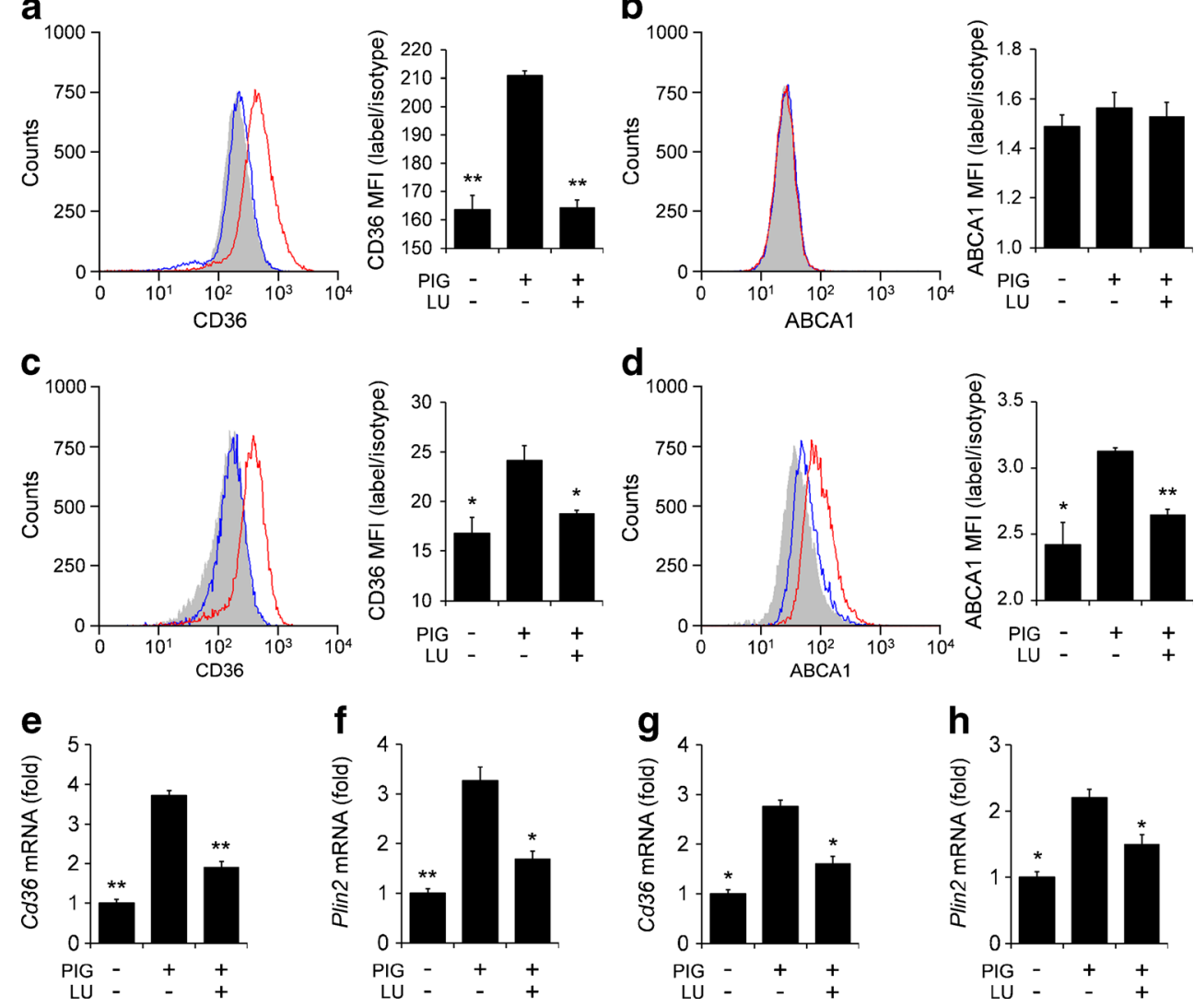
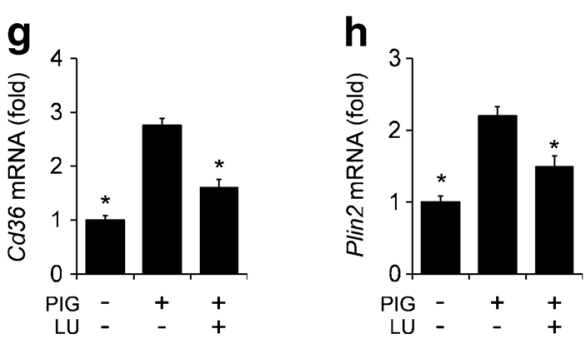
Fig. 5 LU activated AMPK $\alpha 1$ signalling in inflammatory macrophages. (a) Dietary LU increased AMPK $\alpha 1$ activity in EATs in HFD-fed mice. Black bars, HFD; white bars, HFD + LU20; grey bars, HFD + LU10; hatched bars, LFD. $n=8$ per group, one-way ANOVA with Duncan's post hoc test relative to HFD; ${ }^{*} p<0.05,{ }^{* *} p<0.01$. Immunoblots for phosphoAMPK $\alpha 1$ and total AMPK $\alpha 1$ and quantification of phosphorylated AMPK $\alpha 1$ to total AMPK $\alpha 1$ in LPS-treated (b) or PIG-treated (c) RAW264.7 cells and PCMs. C.C, compound C. Data are presented as means \pm SEM, $n=4$ per group, two-tailed Student's $t$ test; ${ }^{*} p<0.05$, $* * p<0.01$
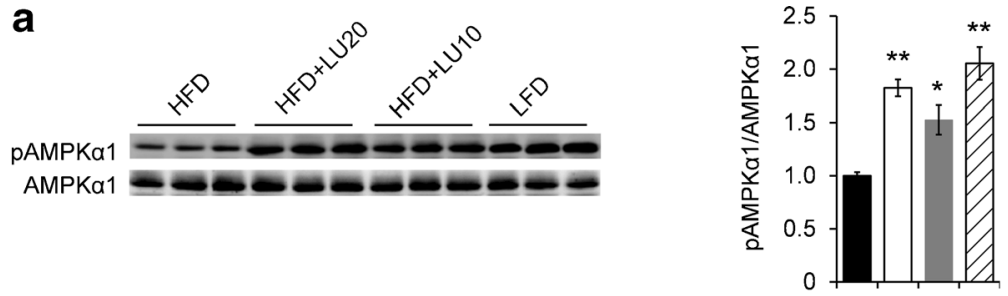

b

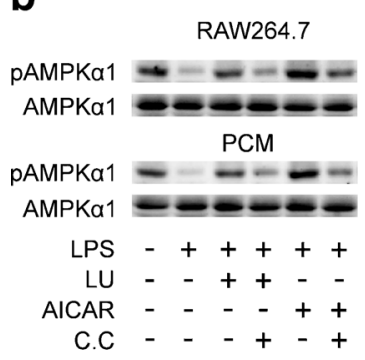

C

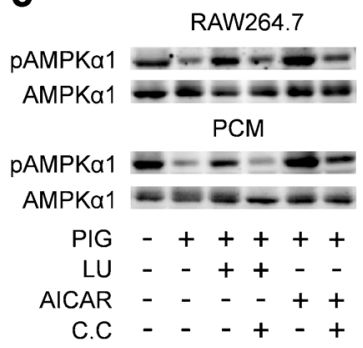

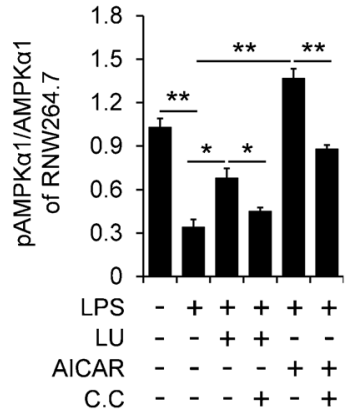
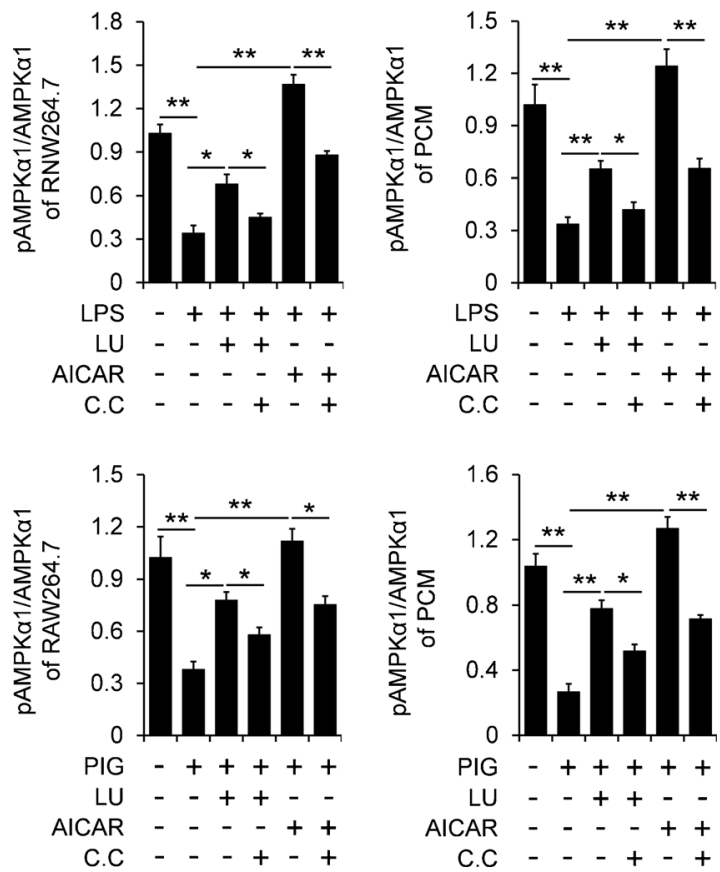

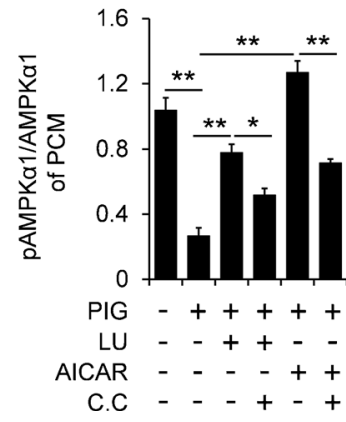

(Fig. 6e-h), were also turned back by LU and AICAR treatment. Finally, compound C treatment (Fig. 6a-h, ESM Fig. 5) and Ampk 1 silencing (Fig. 6i-p, ESM Figs 6, 7) could abrogate the actions of LU.

\section{LU treatment ameliorated insulin sensitivity in} CM-simulated 3T3-L1 adipocytes by activating AMPK signalling RAW264.7 and PCM CM significantly reduced the ratio of p-Akt to total Akt in differentiated 3T3-L1 adipocytes (Fig. 7). LU pretreatment of macrophages blunted the decline of Akt phosphorylation (Fig. 7). Furthermore, compound $\mathrm{C}$ or Ampka1 silencing abolished the actions of LU (Fig. 7).

\section{Discussion}

This study demonstrates that dietary LU can not only interfere with the establishment of HFD-induced insulin resistance, but also ameliorate pre-established insulin resistance and adipose tissue inflammation. Furthermore, LU antagonised M1 or MMe macrophage polarisation in EATs in HFD-fed mice. In addition, in vitro and in vivo studies showed that LU directly suppressed inflammatory macrophage polarisation by activating AMPK $\alpha 1$ signalling. These findings suggest that dietary LU reduces obesity-associated insulin resistance by activating AMPK $\alpha 1$ signalling in ATMs in HFD-fed mice.

LU possesses various bioactivities, including antiinflammatory, anti-cancer and anti-oxidation activities $[29,30]$. In rat models of streptozotocin-induced type 1 diabetes, $\mathrm{LU}$ administration has been reported to reduce blood glucose levels [31] and ameliorate diabetic cardiomyopathy [32] and nephropathy [31], suggesting its potency in improving glucose and lipid metabolism in animals. In addition, LU supplementation in mice fed with an HFD during the establishment of DIO has previously been reported to protect the animals from HFD-induced insulin resistance [15]. However, the effects of LU on pre-established insulin resistance in HFD-fed mice remain unclear. In the current study, we established insulin resistance with a 10-week HFD (ESM Fig. 1), and then supplemented the HFD with LU. Compared with the HFD group, the HFD + LU10 group showed significantly higher glucose tolerance and insulin sensitivity (Fig. 1b-d). Our results reveal that dietary LU not only resisted the establishment of HFD-induced insulin resistance, but also reversed pre-established insulin resistance.

Visceral adipose tissue inflammation critically promotes local and systemic insulin resistance [5, 33]. Various immune 
Fig. 6 LU blocked macrophage inflammatory polarisation through activating AMPK $\alpha 1$ signalling. mRNA levels of the M1 marker Nos2 and the M2 marker Arg1 were detected in LPS-stimulated RAW264.7 cells $(\mathbf{a}, \mathbf{b})$ and PCMs (c, d). mRNA levels of the MMe markers $C d 36$ and Plin2 in PIG-treated RAW264.7 cells (e, f) and PCMs $(\mathbf{g}, \mathbf{h})$. Amp $k$ siRNA abrogated the actions of LU on the mRNA expression of Nos 2 and Arg1 in LPS-stimulated RAW264.7 cells $(\mathbf{i}, \mathbf{j})$ and PCMs $(\mathbf{k}, \mathbf{l})$, and of MMe marker genes in PIGtreated RAW264.7 cells $(\mathbf{m}, \mathbf{n})$ and PCMs $(\mathbf{o}, \mathbf{p})$. All data are presented as means \pm SEM, $n=4$ per group, two-tailed Student's $t$ test; ${ }^{*} p<0.05, * * p<0.01$. C.C, compound C. Con, control
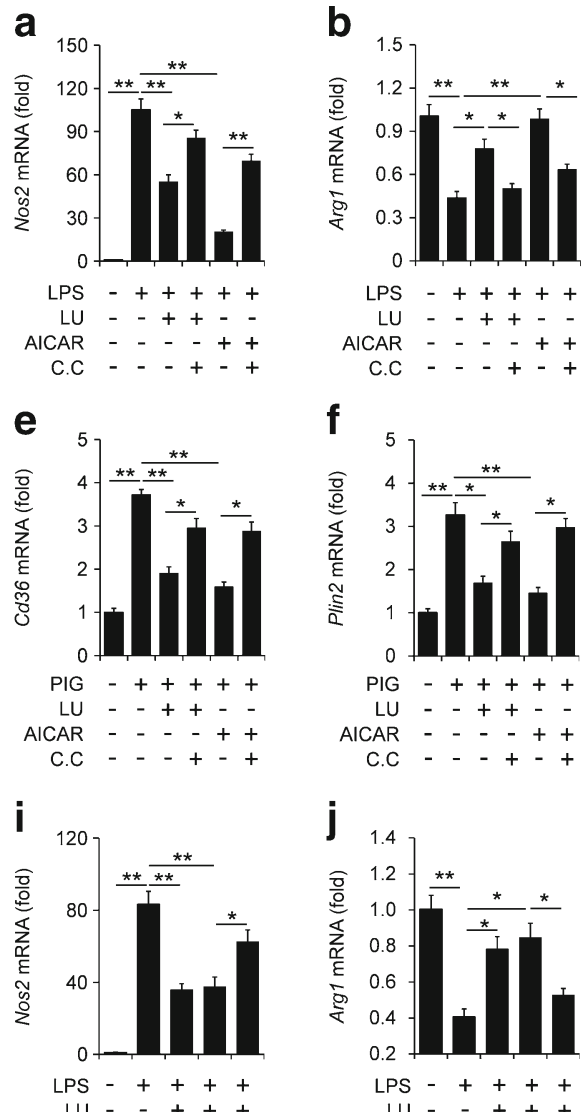

Con siRNA $-\quad-\quad+\quad+$ Ampka1 siRNA - - - - +

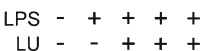
Con SiRNA - - - + Ampka1 siRNA
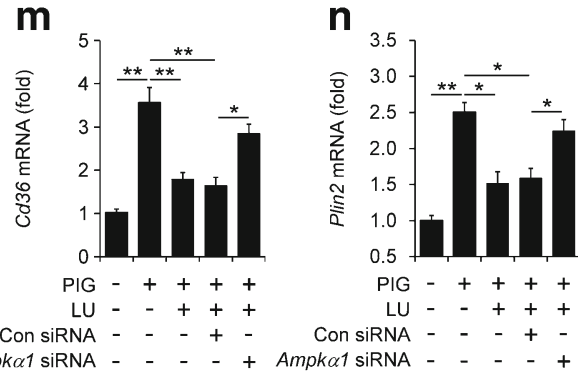
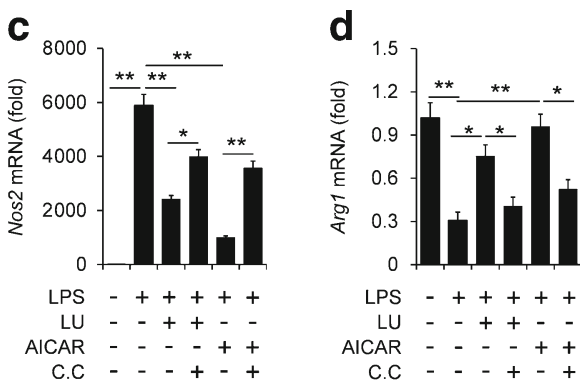

g


Ampk $\alpha 1$ siRNA - - -++ Ampk $\alpha 1$ siRNA - $-{ }_{-}+$



cells, including macrophages, mast cells, neutrophils, eosinophils and $\mathrm{B}$ and $\mathrm{T}$ cells, have been implicated in inflammatory regulation of adipose tissues [5]. In the immune cells, macrophage infiltration and polarisation primarily contribute to adipose tissue inflammation and insulin resistance [7, 8]. Furthermore, other immune cells can affect adipose tissue inflammation by regulating macrophage polarisation [5, 34]. Moreover, in DIO mice, EATs undergo greater macrophage recruitment than other visceral and subcutaneous adipose tissues [9]. Therefore, we proposed that dietary LU should reduce macrophage inflammation and polarisation in EATs in HFD-fed mice.

Our results support this hypothesis. First, mice in the HFD + LU10 and HFD + LU20 groups had lower expression of inflammatory cytokines in EATs than mice in the HFD group
(Fig. 1e). Second, dietary LU reduced macrophage recruitment into EATs in HFD-fed mice (Fig. 2a, ESM Fig. 3). Third, the M1/M2 ratio was modified in EATs in mice fed with an LU-containing HFD (Fig. 2a-d). Finally, dietary LU also downregulated expression of the MMe markers $C d 36$ and Plin2 in EATs (Fig. 2e, f). In conclusion, inflammatory macrophage polarisation, including $\mathrm{M} 1$ and $\mathrm{MMe}$ polarisation, was inhibited by dietary LU in EATs.

Since other immune cells can regulate macrophage polarisation, the direct effects of LU on metabolic dysfunction-associated macrophage polarisation should be investigated. The ATM phenotype undergoes intricate alterations during the development of obesity. LPS stimulation is a classical method of promoting M1 polarisation in vitro [35]. However, the surface markers of LPS-induced M1 
a

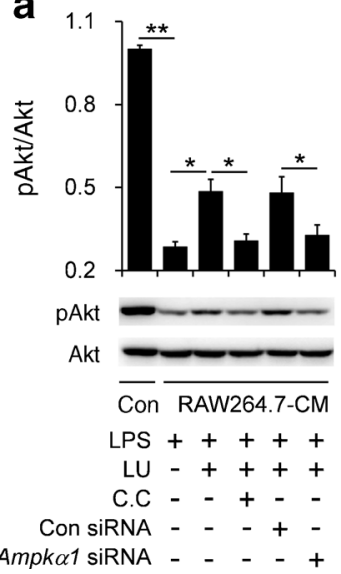

b

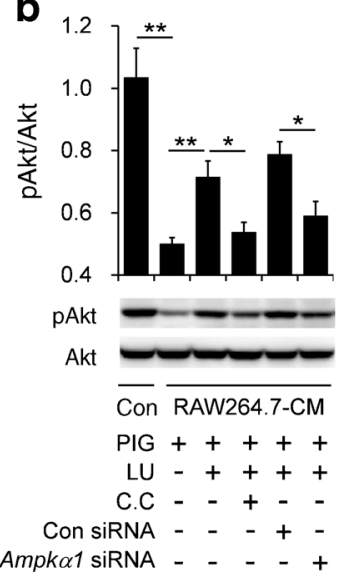

Fig. 7 LU enhanced insulin sensitivity in CM-stimulated 3T3-L1 adipocytes by activating macrophage AMPK $\alpha 1$ signalling. Immunoblots for phospho-Akt and total Akt were performed in 3T3-L1 adipocytes that

macrophages are distinct from those of inflammatory ATMs in obese humans and mice [14]. Moreover, LPS stimulation also suppresses the expression of CD11c, which is the most common marker of M1 macrophages in adipose tissue [36]. Therefore, in addition to LPS-stimulated macrophages, we need to use other obesity-associated macrophage phenotypes. Relative to LPS, metabolic-disease-regulated molecules such as glucose, insulin and palmitate induce an inflammatory MMe phenotype. The markers of the MMe subtype, including CD36, ABCA1 and PLIN2, are more similar to ATMs in obese individuals [14]. Thus, we used LPS- and PIG-induced macrophages to further assess whether LU can directly regulate ATM polarisation. We established both LPS-induced (Fig. 3, ESM Fig. 4) and PIG-induced (Fig. 4, ESM Fig. 4) inflammatory macrophage phenotypes in the commercial RAW264.7 cell line and primary cultured PCMs. LU treatment remarkably inhibited inflammatory cytokine expression (ESM Fig. 4) and reversed alterations in M1, M2 and MMe markers in the cells (Figs 3, 4). These results indicate that LU treatment directly suppresses obesity-associated macrophage polarisation, and thereby maintains insulin sensitivity in HFD-fed mice.

AMPK $\alpha 1$ plays an important role in the suppression of proinflammatory responses and macrophage inflammatory polarisation [21, 22]. Accompanied by improvements in insulin resistance and ATM inflammation, mice in the HFD + LU10 and HFD + LU20 groups also showed increased EAT AMPK $\alpha 1$ signalling (Fig. 5a). Furthermore, LU treatment blocked LPS- and PIG-induced inactivation of AMPK $\alpha 1$ (Fig. 5b, c) and reserved the polarised alterations (Fig. 6) in RAW264.7 cells and PCMs. Moreover, both selective AMPK inhibitor compound $\mathrm{C}$ and Ampkol silencing could abolish the effects of LU on inflammatory macrophage polarisation and associated insulin signalling in adipocytes (Figs 5, 6, 7, ESM Figs 5-7). These results suggest that AMPK $\alpha 1$ is
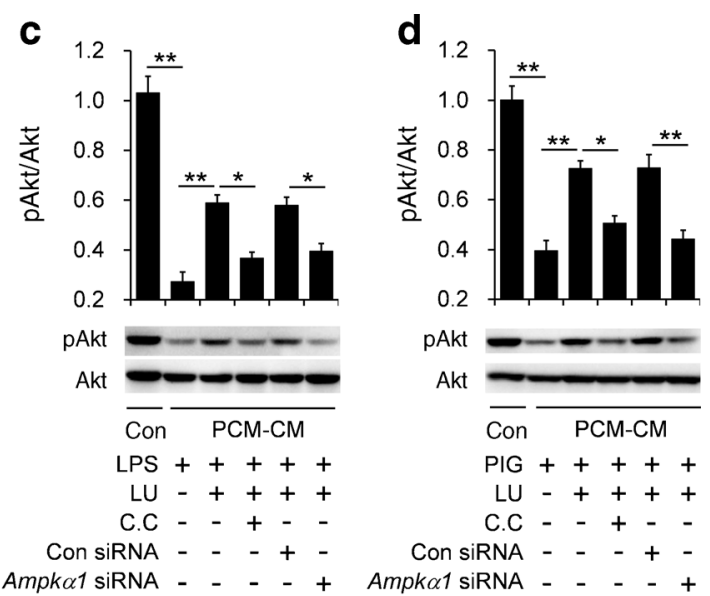

were stimulated with CM from RAW264.7 cells (a, b) or PCMs (c, d). All data are presented as means \pm SEM, $n=4$ per group, two-tailed Student's $t$ test; ${ }^{*} p<0.05,{ }^{*} p<0.01$. C.C, compound C; Con, control

critically involved in LU-reducing ATM polarisation and insulin resistance in HFD-fed mice, although the detailed molecular mechanisms behind macrophage AMPK $\alpha 1$ activation by LU need further investigation.

Although we found, in the current study, that LU treatment could abate inflammatory macrophage polarisation and reduce $\mathrm{CM}$-induced insulin resistance by activating AMPK $\alpha 1$, the improvements in insulin resistance in HFD-fed mice might not be solely attributed to the inhibition of ATM polarisation and inflammation by LU. It has previously been reported that LU can activate AMPK signalling in differentiated 3T3-L1 adipocytes [24] and HepG2 hepatocytes [23]. In addition, AMPK, as an important nutrient sensor, can promote fatty acid oxidation [37] and energy expenditure [38]. Indeed, in the HFD + LU20 group, dietary LU not only suppressed EAT macrophage polarisation (Fig. 2), but also reduced body and subcutaneous adipose tissue weights (ESM Fig. 2). Therefore, LU might also enhance adipocyte AMPK signalling and associated fatty acid oxidation and energy expenditure in HFD-fed mice, a hypothesis that merits further investigation.

In conclusion, dietary LU inhibits ATM inflammatory polarisation in HFD-fed mice, suggesting its potential in remedying and intervening in common metabolic disorders.

Funding This work was supported by grants from the National Natural Science Foundation of China (31171315) to JL; The Fundamental Research Funds for Central Universities, China (2014HGCH0005), to JL; Anhui Science and Technology Research Projects of China (1401b042018) to JL; Anhui Provincial Natural Science Foundation of China (1408085QC48) to BB; and Anhui Provincial Natural Science Foundation of China (1508085MC58) to WQ.

Duality of interest The authors declare that there is no duality of interest associated with this manuscript. 
Contribution statement $\mathrm{LZ}$ and JL conceived and designed the study. $\mathrm{LZ}$ and $\mathrm{YH}$ acquired, analysed and interpreted data and revised the article critically for important intellectual content. XW and XZ analysed and interpreted data and revised the article critically for important intellectual content. BB and WQ interpreted data and revised the article critically for important intellectual content. $\mathrm{LZ}$ and JL analysed and interpreted data and drafted and revised the article. JL has primary responsibility for the final content. All authors read and approved the final manuscript.

\section{References}

1. Huh JY, Park YJ, Ham M, Kim JB (2014) Crosstalk between adipocytes and immune cells in adipose tissue inflammation and metabolic dysregulation in obesity. Mol Cells 37:365-371

2. Lee BC, Lee J (2014) Cellular and molecular players in adipose tissue inflammation in the development of obesity-induced insulin resistance. Biochim Biophys Acta 1842:446-462

3. Gregor MF, Hotamisligil GS (2011) Inflammatory mechanisms in obesity. Annu Rev Immunol 29:415-445

4. Ouchi N, Parker JL, Lugus JJ, Walsh K (2011) Adipokines in inflammation and metabolic disease. Nat Rev Immunol 11:85-97

5. Mathis D (2013) Immunological goings-on in visceral adipose tissue. Cell Metab 17:851-859

6. Despres JP, Lemieux I (2006) Abdominal obesity and metabolic syndrome. Nature 444:881-887

7. Weisberg SP, McCann D, Desai M, Rosenbaum M, Leibel RL, Ferrante AW Jr (2003) Obesity is associated with macrophage accumulation in adipose tissue. J Clin Invest 112:1796-1808

8. Xu JM, Shi GP (2012) Emerging role of mast cells and macrophages in cardiovascular and metabolic diseases. Endocr Rev 33:71-108

9. Altintas MM, Azad A, Nayer B et al (2011) Mast cells, macrophages, and crown-like structures distinguish subcutaneous from visceral fat in mice. J Lipid Res 52:480-488

10. Lumeng CN, Bodzin JL, Saltiel AR (2007) Obesity induces a phenotypic switch in adipose tissue macrophage polarization. J Clin Invest 117:175-184

11. Wentworth JM, Naselli G, Brown WA et al (2010) Proinflammatory $\mathrm{CD} 11 \mathrm{c}^{+} \mathrm{CD} 206^{+}$adipose tissue macrophages are associated with insulin resistance in human obesity. Diabetes 59: $1648-1656$

12. Lumeng CN, DelProposto JB, Westcott DJ, Saltiel AR (2008) Phenotypic switching of adipose tissue macrophages with obesity is generated by spatiotemporal differences in macrophage subtypes. Diabetes 57:3239-3246

13. Chmelar J, Chung KJ, Chavakis T (2013) The role of innate immune cells in obese adipose tissue inflammation and development of insulin resistance. Thromb Haemost 109:399-406

14. Kratz M, Coats BR, Hisert KB et al (2014) Metabolic dysfunction drives a mechanistically distinct proinflammatory phenotype in adipose tissue macrophages. Cell Metab 20:614-625

15. Xu N, Zhang L, Dong J et al (2014) Low-dose diet supplement of a natural flavonoid, luteolin, ameliorates diet-induced obesity and insulin resistance in mice. Mol Nutr Food Res 58:1258-1268

16. Wu W, Li D, Zong Y et al (2013) Luteolin inhibits inflammatory responses via p38/MK2/TTP-mediated mRNA stability. Molecules 18:8083-8094

17. Ando C, Takahashi N, Hirai S et al (2009) Luteolin, a food-derived flavonoid, suppresses adipocyte-dependent activation of macrophages by inhibiting JNK activation. FEBS Lett 583:3649-3654

18. Chen CY, Peng WH, Tsai KD, Hsu SL (2007) Luteolin suppresses inflammation-associated gene expression by blocking NF-kB and AP-1 activation pathway in mouse alveolar macrophages. Life Sci $81: 1602-1614$
19. Steinberg GR, Schertzer JD (2014) AMPK promotes macrophage fatty acid oxidative metabolism to mitigate inflammation: implications for diabetes and cardiovascular disease. Immunol Cell Biol 92:340-345

20. Salminen A, Hyttinen JM, Kaarniranta K (2011) AMP-activated protein kinase inhibits NF-kB signaling and inflammation: impact on healthspan and lifespan. J Mol Med (Berl) 89:667-676

21. Sag D, Carling D, Stout RD, Suttles J (2008) Adenosine 5'monophosphate-activated protein kinase promotes macrophage polarization to an anti-inflammatory functional phenotype. J Immunol 181:8633-8641

22. Galic S, Fullerton MD, Schertzer JD et al (2011) Hematopoietic AMPK $\beta 1$ reduces mouse adipose tissue macrophage inflammation and insulin resistance in obesity. J Clin Invest 121:4903-4915

23. Liu JF, Ma Y, Wang Y, Du ZY, Shen JK, Peng HL (2011) Reduction of lipid accumulation in HepG2 cells by luteolin is associated with activation of AMPK and mitigation of oxidative stress. Phytother Res 25:588-596

24. Xiao N, Mei F, Sun Y, Pan G, Liu B, Liu K (2014) Quercetin, luteolin, and epigallocatechin gallate promote glucose disposal in adipocytes with regulation of AMP-activated kinase and/or sirtuin 1 activity. Planta Med 80:993-1000

25. Dong J, Zhang X, Zhang L et al (2014) Quercetin reduces obesityassociated ATM infiltration and inflammation in mice: a mechanism including AMPK $\alpha 1 /$ SIRT1. J Lipid Res 55:363-374

26. Bao B, Chen YG, Zhang L et al (2013) Momordica charantia (bitter melon) reduces obesity-associated macrophage and mast cell infiltration as well as inflammatory cytokine expression in adipose tissues. PLoS One 8:e84075

27. Ceppo F, Berthou F, Jager J, Dumas K, Cormont M, Tanti JF (2014) Implication of the Tpl2 kinase in inflammatory changes and insulin resistance induced by the interaction between adipocytes and macrophages. Endocrinology 155:951-964

28. Kim D, Kim J, Yoon JH et al (2014) CXCL12 secreted from adipose tissue recruits macrophages and induces insulin resistance in mice. Diabetologia 57:1456-1465

29. Lopez-Lazaro M (2009) Distribution and biological activities of the flavonoid luteolin. Mini Rev Med Chem 9:31-59

30. Harris GK, Qian Y, Leonard SS, Sbarra DC, Shi X (2006) Luteolin and chrysin differentially inhibit cyclooxygenase-2 expression and scavenge reactive oxygen species but similarly inhibit prostaglandin-E2 formation in RAW 264.7 cells. J Nutr 136: $1517-1521$

31. Wang GG, Lu XH, Li W, Zhao X, Zhang C (2011) Protective effects of luteolin on diabetic nephropathy in STZ-induced diabetic rats. Evid Based Complement Alternat Med 2011:323171

32. Wang G, Li W, Lu X, Bao P, Zhao X (2012) Luteolin ameliorates cardiac failure in type I diabetic cardiomyopathy. J Diabetes Complications 26:259-265

33. Donath MY, Shoelson SE (2011) Type 2 diabetes as an inflammatory disease. Nat Rev Immunol 11:98-107

34. Zhou Y, Yu X, Chen H et al (2015) Leptin deficiency shifts mast cells toward anti-inflammatory actions and protects mice from obesity and diabetes by polarizing M2 macrophages. Cell Metab 22: $1045-1058$

35. Martinez FO, Sica A, Mantovani A, Locati M (2008) Macrophage activation and polarization. Front Biosci 13:453-461

36. Becker L, Liu NC, Averill MM et al (2012) Unique proteomic signatures distinguish macrophages and dendritic cells. PLoS One 7:e33297

37. Herms A, Bosch M, Reddy BJ et al (2015) AMPK activation promotes lipid droplet dispersion on detyrosinated microtubules to increase mitochondrial fatty acid oxidation. Nat Commun 6:7176

38. Canto C, Gerhart-Hines Z, Feige JN et al (2009) AMPK regulates energy expenditure by modulating $\mathrm{NAD}^{+}$metabolism and SIRT1 activity. Nature 458:1056-1060 